\section{Mathematical Modeling in Biology}

\subsection{Introduction}

Mathematics permeates biology. Unfortunately, this is far from obvious to most students of biology. While many biology courses cover results and insights from mathematical models, they rarely describe how these results were obtained. Typically, it is only when biologists start reading research articles that they come to appreciate just how common mathematical modeling is in biology. For many students, this realization comes long after they have chosen the majority of their courses, making it difficult to build the mathematical background needed to appreciate and feel comfortable with the mathematics that they encounter. This book is a guide to help any student develop this appreciation and comfort. To motivate learning more mathematics, we devote this first chapter to emphasizing just how common mathematical models are in biology and to highlighting some of the important ways in which mathematics has shaped our understanding of biology.

Let's begin with some numbers. According to BIOSIS, 886,101 articles published in biological journals contain the keyword "math" (including math, mathematical, mathematics, etc.) as of April 2006. Some of these articles are in specialized journals in mathematical biology, such as the Bulletin of Mathematical Biology, the Journal of Mathematical Biology, Mathematical Biosciences, and Theoretical Population Biology. Many others, however, are published in the most prestigious journals in science, including Nature and Science. Such a coarse survey, however, misses a large fraction of articles describing theoretical models without using "math" as a keyword.

We performed a more in-depth survey of all of the articles published in one year within some popular ecology and evolution journals (Table 1.1). Given that virtually every statistical analysis is based on an underlying mathematical model, nearly all articles relied on mathematics to some extent. With a stricter definition that excludes papers whose only use of mathematics is through statistical analyses, 35\% of Evolution and Ecology articles and nearly $60 \%$ of American Naturalist articles reported predictions or results obtained using mathematical models. The extent of mathematical analysis varied greatly, but mathematical equations appeared in almost all of these articles. Furthermore, many of the articles used computer simulations to describe changes that occur over time in the populations under study. Such simulations can be incredibly helpful, allowing the reader to "see" what the equations predict and allowing authors to obtain results from even the most complicated models.
Chapter Goals:

- To develop an appreciation for the importance of mathematics in biology

Chapter Concepts:

- Variables

- Dynamics

- Parameters

- Principle of parsimony 
(C) Copyright, Princeton University Press. No part of this book may be distributed, posted, or reproduced in any form by digital or mechanical means without prior written permission of the publisher.

TABLE 1.1

Use of mathematical models in full-length journal articles

\begin{tabular}{lcccc}
\hline Journal (in 2001) & $\begin{array}{l}\text { Number of } \\
\text { articles }\end{array}$ & $\begin{array}{l}\text { General use } \\
\text { of models }^{\mathrm{a}}\end{array}$ & $\begin{array}{l}\text { Specific use } \\
\text { of models }^{\mathrm{b}}\end{array}$ & $\begin{array}{c}\text { Equations } \\
\text { presented }^{\mathrm{c}}\end{array}$ \\
\hline American Naturalist & 105 & $96 \%$ & $59 \%$ & $58 \%$ \\
Ecology & 274 & $100 \%$ & $35 \%$ & $38 \%$ \\
Evolution & 231 & $100 \%$ & $35 \%$ & $33 \%$ \\
\hline
\end{tabular}

${ }^{\mathrm{a}}$ General use: Used a mathematical model in the broadest sense, including statistical or phylogenetic analyses with a mathematical basis (e.g., ANOVA, regression, etc.).

bSpecific use: Used a mathematical model to obtain results (excluding cases that involve only statistical or phylogenetic analyses); the model may or may not be derived in the paper.

'Equations presented: Excluding standard statistical equations.

An important motivation for learning mathematical biology is that mathematical equations typically "say" more than the surrounding text. Given the space constraints of many journals, authors often leave out intermediate steps or fail to state every assumption that they have made. Being able to read and interpret mathematical equations is therefore extremely important, both to verify the conclusions of an author and to evaluate the limitations of unstated assumptions.

To describe all of the biological insights that have come from mathematical models would be an impossible task. Therefore, we focus the rest of this chapter on the insights obtained from mathematical models in one tiny, but critically important, area of biology: the ecology and epidemiology of the human immunodeficiency virus (HIV). As we shall see, mathematical models have allowed biologists to understand otherwise hidden aspects of HIV, they have produced testable predictions about how HIV replicates and spreads, and they have generated forecasts that improve the efficacy of prevention and health care programs.

\subsection{HIV}

On June 5, 1981, the Morbidity and Mortality Weekly Report of the Centers for Disease Control reported the deaths of five males in Los Angeles, all of whom had died from pneumocystis, a form of pneumonia that rarely causes death in individuals with healthy immune systems. Since this first report, acquired immunodeficiency syndrome (AIDS), as the disease has come to be known, has reached epidemic proportions, having caused more than 20 million deaths worldwide (Joint United Nations Programme on HIV/AIDS 2004b). AIDS results from the deterioration of the immune system, which then fails to ward off various cancers (e.g., Karposi's sarcoma) and infectious agents (e.g., the protozoa that cause pneumocystis, the viruses that cause retinitis, and the bacteria that cause tuberculosis). The collapse of the immune system is caused by infection with the human immunodeficiency virus (Figure 1.1). HIV is transmitted 


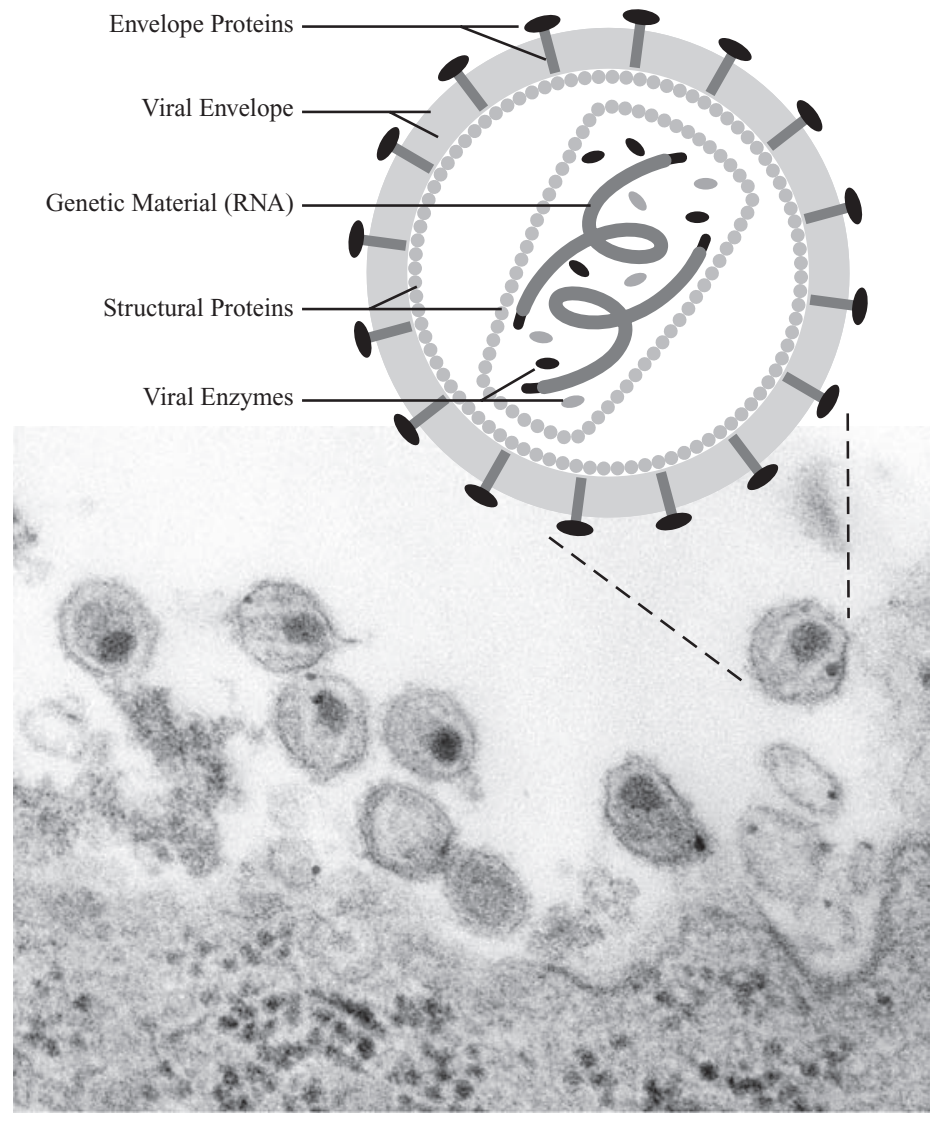

Figure 1.1: The human immunodeficiency virus. Electron micrograph shows HIV co-cultivated with human lymphocytes (courtesy of CDC; A. Harrison, P. Feorino, and E. L. Palmer).

from infected to susceptible individuals by the exchange of bodily fluids, primarily through sexual intercourse without condoms, sharing of unsterilized needles, or transfusion with infected blood supplies (although routine testing for HIV in donated blood has reduced the risk of infection through blood transfusion from 1 in 2500 to 1 in 250,000 [Revelle 1995]).

Once inside the body, HIV particles infect white blood cells by attaching to the CD4 protein embedded in the cell membranes of helper $\mathrm{T}$ cells, macrophages, and dendritic cells. The genome of the virus, which is made up of RNA, then enters these cells and is reverse transcribed into DNA, which is subsequently incorporated into the genome of the host. (The fact that normal transcription from DNA to RNA is reversed is why HIV is called a retrovirus.) The virus may then remain latent within the genome of the host cell or become activated, in which case it is transcribed to produce both the proteins necessary to replicate and daughter RNA particles (Figure 1.2). When actively replicating, HIV can produce hundreds of daughter viruses per day per host cell (Dimitrov et al. 1993), often killing the host cell in the process. These virus particles (or virions) then go on to infect other CD4-bearing cells, repeating the process. Eventually, without treatment, the population of CD4+ helper T cells declines dramatically from about 1000 cells per cubic millimeter of blood to about 200 cells, signaling the onset of AIDS (Figure 1.3). 


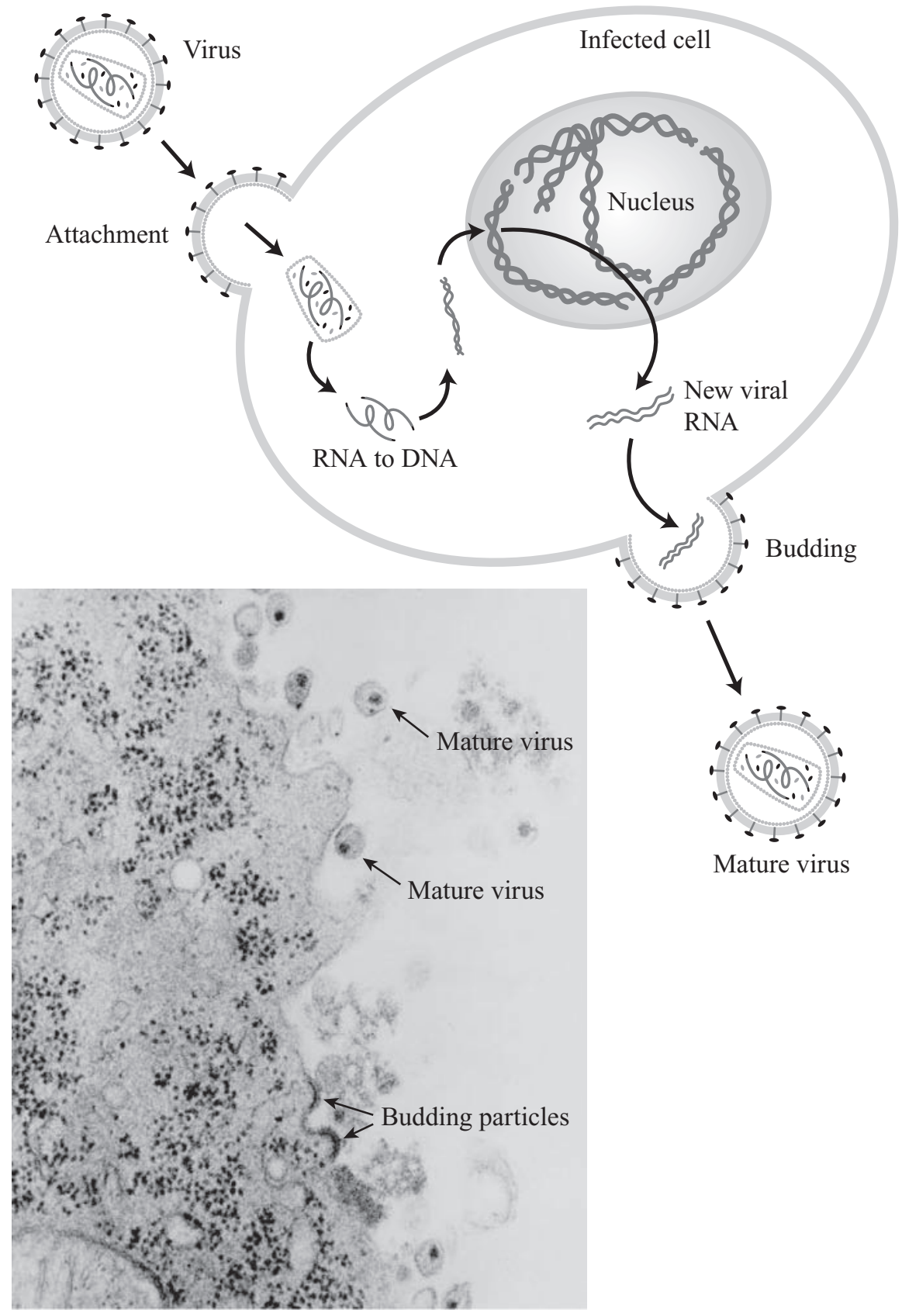

Figure 1.2: The life cycle of HIV within a host cell. Electron micrograph shows budding and mature HIV (courtesy of CDC; A. Harrison, E. L. Palmer, and P. Feorino).

Normally, CD4+ helper T cells function in the cellular immune response by binding to fragments of viruses and other foreign proteins presented on the surface of other immune cells. This binding activates the helper $\mathrm{T}$ cells to release chemicals (cytokines), which stimulate both killer T cells to attack the infected cells and B cells to manufacture antibodies against the foreign particles. What makes HIV particularly harmful to the immune system is that the 


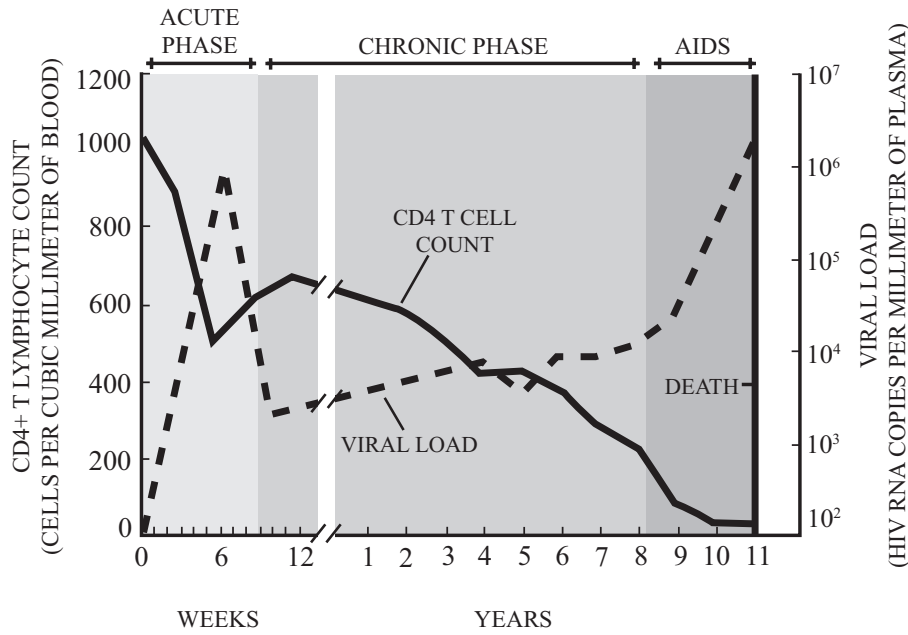

Figure 1.3: The time course of HIV infection within an individual. Viral loads and CD4 + T cell counts are plotted over time since infection. Based on data in Fauci et al. (1996).

virus preferentially attacks activated helper T cells; by destroying such cells, HIV can eliminate the very cells that recognize and fight other infections.

Early on in the epidemic, the median period between infection with HIV-1 (the strain most common in North America) and the onset of AIDS was about ten years (Bacchetti and Moss 1989). The median survival time following the onset of an AIDS-associated condition (e.g., Karposi's sarcoma or pneumocystis) was just under one year (Bacchetti et al. 1988). Survival statistics have improved dramatically with the development of effective antiretroviral therapies, such as protease inhibitors, which first became available in 1995, and with the advent of combination drug therapy, which uses multiple drugs to target different steps in the replication cycle of HIV. In San Francisco, the median survival after diagnosis with an AIDS-related opportunistic infection rose from 17 months between 1990 and 1994 to 59 months between 1995 and 1998 (San Francisco Department of Public Health 2000). Unfortunately, modern drug therapies are extremely expensive (typically over US\$10,000 per patient per year) and cannot be afforded by the majority of individuals infected with HIV worldwide. Until effective therapy or vaccines become freely available, HIV will continue to take a devastating toll (Figure 1.4; Joint United Nations Programme on HIV/AIDS 2004a).

\subsection{Models of HIV/AIDS}

Mathematical modeling has been a very important tool in HIV/AIDS research. Every aspect of the natural history, treatment, and prevention of HIV has been the subject of mathematical models, from the thermodynamic characteristics of HIV (e.g., Hansson and Aqvist 1995; Kroeger Smith et al. 1995; Markgren et al. 2001) to its replication rate both within and among individuals (e.g., Funk et al. 2001; Jacquez et al. 1994; Koopman et al. 1997; Levin et al. 1996; Lloyd 2001; Phillips 1996). In the following sections, we describe four of these models in more detail. These models were chosen because of their implications 


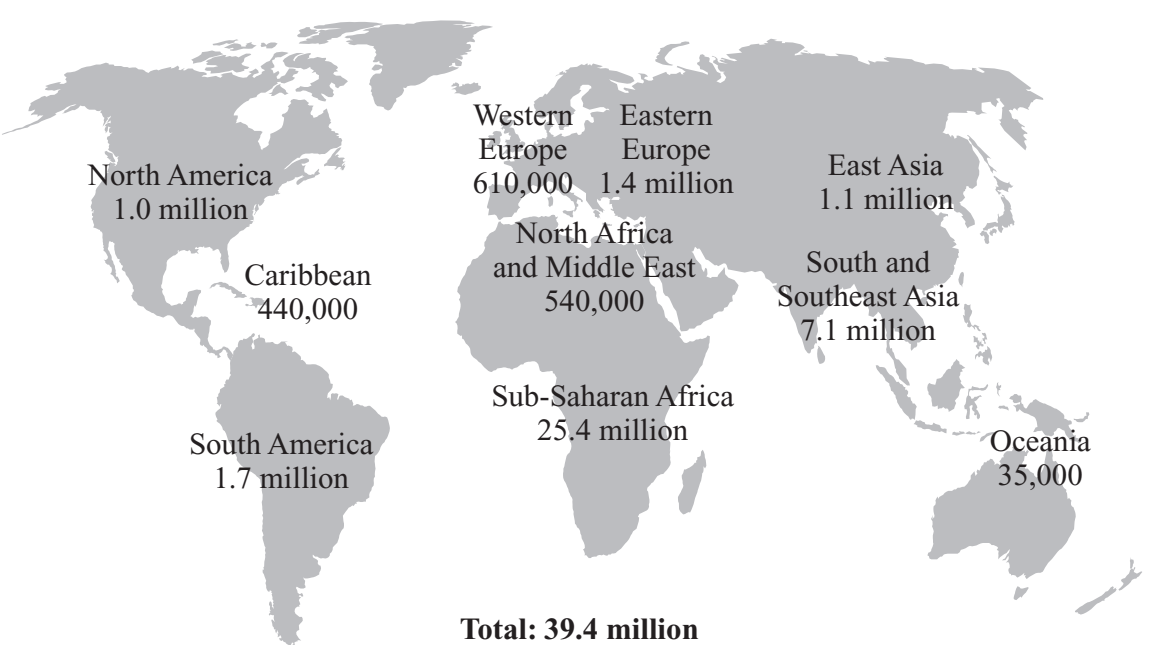

Figure 1.4: Number of individuals living with HIV. The number of adults and children estimated to be living with HIV is shown (Joint United Nations Programme on HIV/AIDS, 2004a).

for our understanding of HIV, but they also illustrate the sorts of techniques that are described in the rest of this book.

A variable of a model is a quantity that changes over time.

The dynamics of a system is the pattern of changes that occur over time.

\subsubsection{Dynamics of HIV after Initial Infection}

After an individual is infected by HIV, the number of virions within the bloodstream skyrockets and then plummets again (Figure 1.3). This period of primary HIV infection is known as the acute phase; it lasts approximately 100 days and often leads to the onset of flu-like symptoms (Perrin and Yerly 1997; Schacker et al. 1996). The rapid rise in virus particles reflects the infection of CD4 + cells and the replication of HIV within actively infected host cells. But what causes the decline in virus particles? The most obvious answer is that the immune system acts to recognize and suppress the viral infection (Koup et al. 1994). Phillips (1996), however, suggested an alternative explanation: the number of virions might decline because most of the susceptible CD4+ cells have already been infected and thus there are fewer host cells to infect. Phillips developed a model to assess whether this alternative explanation could mimic the observed rise and fall of virions in the blood stream over the right time frame. In his model, there are four variables (i.e., four quantities that change over time): $R, L, E$, and $V . R$ represents the number of activated but uninfected CD4 + cells, $L$ represents the number of latently infected cells, $E$ represents the number of actively infected cells, and $V$ represents the number of virions in the blood stream. The dynamics of each variable (i.e., how the variable changes over time) depend on the values of the remaining variables. For example, the number of viruses changes over time in a manner that depends on the number of cells infected with actively replicating HIV. In the next chapter, we describe the steps involved in building models such as this one (see Chapter 2, Box 2.4).

Phillips' model contains several parameters, which are quantities that are constant over time (see Chapter 2, Box 2.4). In particular, the death rate of 


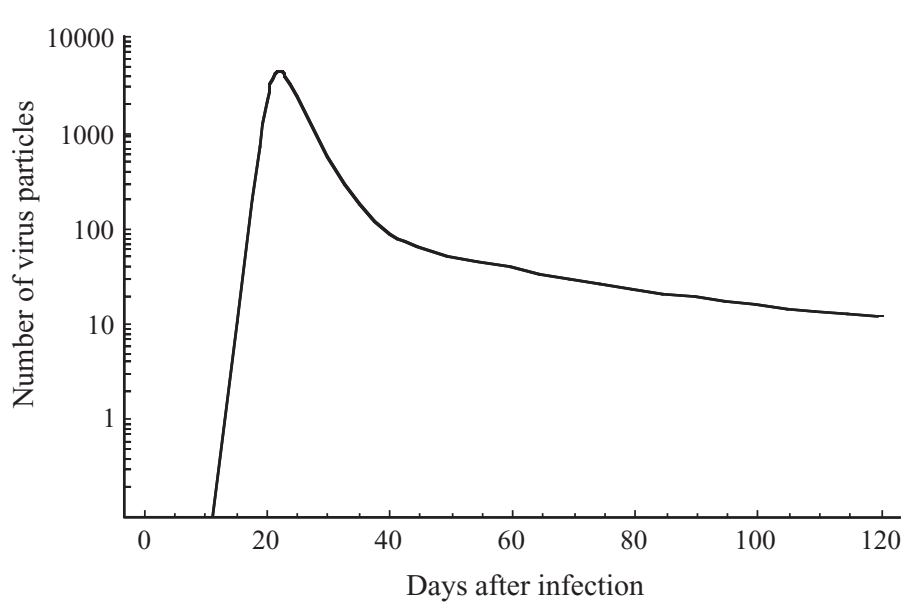

Figure 1.5: Number of virus particles in the blood stream. Based on the model and parameter values of Phillips (1996), the number of virions per $\mathrm{mm}^{3}$ blood $(V)$ is shown as a function of the number of days since primary infection ( $y$-axis is plotted on a log-scale). Around a month after infection, the number of virus particles declines by about 100 -fold even without any specific response by the immune system. See Box 2.4 in Chapter 2 for more details. actively infected cells $(\delta)$ and the death rate of viruses $(\sigma)$ are parameters in the model and are not allowed to change over time. ( $\delta$ and $\sigma$ are the lower-case Greek letters "delta" and "sigma." Greek letters are often used in models, especially for terms that remain constant ("parameters"). See Table 2.1 for a complete list of Greek letters.) Thus, Phillips built into his model the crucial assumption that the body does not get better at eliminating infected cells or virus particles over time, under the null hypothesis that the immune system does not mount a defense against HIV during the acute phase. To model the progression of HIV within the body, Phillips then needed values for each of the parameters in the model. Unfortunately, few data existed at the time for many of them. To proceed, Phillips chose plausible values for each parameter and numerically ran the model (a technique that we will describe in Chapter 4). The numerical solution for the number of virus particles, $V$, predicted from Phillips' model is plotted in Figure 1.5 (compare to Figure 1.3). Phillips then showed that similar patterns are observed under a variety of different parameter values. In particular, he observed that the number of virus particles typically rose and then fell by several orders of magnitude over a period of a few days to weeks. (An order of magnitude refers to a factor of ten. The number 100 is two orders of magnitude larger than one.)

Phillips thus came to the counterintuitive conclusion that "the reduction in virus concentration during acute infection may not reflect the ability of the HIV-specific immune response to control the virus replication" (p. 497, Phillips 1996). The wording of this conclusion is critical and insightful. Phillips did not use his model to prove that the immune system plays no role in viral dynamics during primary infection. In fact, his model cannot say one way or the other whether there is a relevant HIV-specific immune response during this time period. What Phillips can say is that an immune response is not necessary to explain the observed data. This result illustrates an important principle in modeling: the principle of parsimony. The principle of parsimony states that one should prefer models containing as few variables and parameters as possible to describe the essential attributes of a system. Paraphrasing Albert Einstein, a model should be as simple as possible, but no simpler. In Phillips' case, he
A parameter of a model is a quantity that remains constant over time.
According to the principle of parsimony, a simple explanation (or model) should be preferred over a complex explanation if both are equally compatible with the data. 
could have added more variables describing an immune response during acute infection, but his results showed that adding such complexity was unnecessary. A simpler hypothesis can explain the rise and fall of HIV in the bloodstream: as infection proceeds, a decline in susceptible host cells reduces the rate at which virus is produced. Without having a good reason to invoke a more complex model, the principle of parsimony encourages us to stick with simple hypotheses.

Phillips' model accomplished a number of important things. First, it changed our view of what was possible. Without such a model, it would seem unlikely that a dramatic viral peak and decline could be caused by the dynamics of a CD4+ cell population without an immune response. Second, it produced testable predictions. One prediction noted by Phillips is that the viral peak and decline should be observed even in individuals that do not mount an immune response (i.e., do not produce anti-HIV antibodies) over this time period. Indeed, this prediction has been confirmed in several patients (Koup et al. 1994; Phillips 1996). Employing a more quantitative test, Stafford et al. (2000) fitted a version of Phillips' model to data on the viral load in ten patients from several time points during primary HIV infection; they found a good fit to the data within the first 100 days following infection. Third, Phillips' model generated a useful null hypothesis: viral dynamics do not reflect an immune response. This null hypothesis might be wrong, but at least it can be tested.

Phillips acknowledged that this null hypothesis can be rejected as a description of the longer-term dynamics of HIV. His model predicts that the viral load should reach an equilibrium (as described in Chapter 8), but observations indicate that the viral load slowly increases over the long term as the immune system weakens (the chronic phase in Figure 1.3). Furthermore, Schmitz et al. (1999) directly tested Phillips' hypothesis by examining the role of the immune system in rhesus monkeys infected with the simian immunodeficiency virus (SIV), the equivalent of HIV in monkeys. By injecting a particular antibody, Schmitz et al. were able to eliminate most CD8+ lymphocytes, which are the killer T cells thought to prevent the replication of HIV and SIV. Compared to control monkeys, the experimentally treated monkeys showed a much more shallow decline in virus load following the peak. This proves that, at least in monkeys, an immune response does play some role in the viral dynamics observed during primary infection. Nevertheless, the peak viral load was observed at similar levels in antibody-treated and untreated monkeys. Thus, an immune response was not responsible for stalling viral growth during the acute phase, which is best explained, instead, by a decline in the number of uninfected CD4+ cells (the targets of HIV and SIV).

\subsubsection{Replication Rate of HIV}

After the initial acute phase of infection, HIV circulates within the body at low levels until the onset of AIDS (Figure 1.3). These low levels suggest that virus particles might be produced at a low rate per day. This suggestion was, however, shown to be false using mathematical models in conjunction with 
experimental data (Ho et al. 1995; Nowak et al. 1995; Wei et al. 1995). According to the mathematical models, low numbers of virus particles can result from a low rate of viral production, $P$, or from a high rate of clearing virus from the body, $c$ (Ho et al. 1995). Determining which of these possibilities is correct is not possible using only the observed number of virus particles in untreated patients. These landmark papers pointed out, however, that you can tease apart these possibilities using mathematical models that predict viral dynamics following the application of antiretroviral drugs (Ho et al. 1995; Nowak et al. 1995; Wei et al. 1995). For example, Ho et al. (1995) treated HIV-infected patients with ABT-538, an antiviral drug that effectively prevents HIV replication (at least in the short term). Thus, the experimental treatment reduced viral production $P$ to zero, causing the viral load to plummet within the bloodstream. The rate at which the viruses decreased in frequency was consistent with a simple mathematical equation that we will encounter in Chapter 6 (equation (6.10b)). Fitting the mathematical model to the data allowed the authors to obtain an important and surprising result: virus particles were rapidly cleared from the body, with the half-life of HIV in plasma being only a couple of days. The authors thus inferred that the production rate of viruses must normally be enormous, on the order of a billion new viruses produced per day, in order to maintain HIV in the face of high clearance rates. Later work, using more precise experimental data and more detailed modeling, demonstrated that the turnover of HIV is even more rapid, with the half-life of HIV being less than a day and with over 10 billion viruses produced per day. This is a remarkable insight, as it was once thought that relatively little was happening during the chronic phase of HIV infection (Perelson et al. 1996).

These papers had an enormous impact on our understanding of HIV. One of the most important conclusions to follow from this work was that we must expect genetic diversity to be rapidly generated in HIV as a result of the high rate of viral production. If resistance to an antiviral drug requires a particular mutation, it is virtually guaranteed that this mutation will arise rapidly. Only combination drug therapies, requiring multiple mutations for resistance, have a long-term chance of success given the enormous evolutionary potential of HIV.

\subsubsection{The Effects of Antiretroviral Therapy on the Spread of HIV}

The specter of AIDS has softened following the development of effective antiretroviral therapies (ART), involving various drug combinations that have allowed people to live longer with HIV. Public health officials are concerned, however, that this respite will be short lived for two reasons: (a) people may be more inclined to engage in risky behavior knowing that ART exists and (b) HIV might evolve resistance to these drugs, causing the drugs to become ineffective. With these possibilities in mind, Blower et al. (2000) constructed a mathematical model to predict how drug therapy might affect the number of new cases of HIV and the number of deaths due to AIDS. Their model was tailored to data from the San Francisco gay community, where approximately 30\% of men were infected with HIV (HIV+) and approximately $50 \%$ of these were taking combination ART. 
In using their model to predict the future course of HIV, the authors pointed out that uncertainty exists in most model parameters, such as the effect of ART on survival and infectivity, as well as changes in the rate of risky behavior. The authors thus allowed the parameters to be drawn from a range of plausible values rather than assigning one specific number to each. They then drew each parameter from its range and used 1000 different combinations of parameters to predict the spread of HIV. We derive their model in Chapter 2 (Box 2.5) but for the sake of presentation, we simplify it slightly (the model presented by Blower et al. is no more difficult conceptually, it just takes longer to describe). Specifically, we ignore the evolution of resistance by HIV, and we set most parameters to the median value of the range used by Blower et al. (2000). Here, we focus our attention on the parameter describing changes in risky behavior within the gay community following the introduction of ART, and we allow this parameter to take on a range of possible values. Specifically, the average number of sexual partners with whom an uninfected individual (HIV-) has unprotected sex per year is allowed to increase from $c$ before ART by a factor $1+i$. We examine the impact of ART on risk-taking behavior, from causing no change ( $i=0$ ) to doubling the risk $(i=1)$. The variables that are tracked over time are the number of uninfected $(X)$, infected but untreated $\left(Y_{U}\right)$, and infected and treated $\left(Y_{T}\right)$ gay men that are sexually active within San Francisco (see Box 2.5).

This model can be used to address the following questions (Blower et al. 2000): What percentage of AIDS deaths (relative to pre-ART levels) are averted by ART? What percentage of new HIV + cases (relative to pre-ART levels) are averted by ART? How sensitive are these predictions to different choices of parameters, none of which is known with certainty?

The risks that people are willing to take generally depend on the perceived consequences; because antiretroviral therapies have been so effective at countering the progression of HIV and at lowering the infectiousness of HIV, gay men who are sexually active in San Francisco might be more willing to engage in sex without condoms (increasing i). Unfortunately, it is hard to predict exactly how sexual behavior will change. But it is possible, through a model, to investigate a range of possible changes in behavior. Figure 1.6 indicates that the effectiveness of ART depends strongly on these changes in behavior. $43 \%$ of the AIDS deaths that would have occurred within the gay population of San Francisco within the next ten years will be avoided by the use of ART if risky behavior does not increase, but only $24 \%$ of the AIDS deaths will be averted if risky behavior doubles. In fact, if the amount of risky behavior more than triples, then the model predicts more AIDS deaths with drug therapy than without!

A gloomier picture emerges if one looks at the number of new cases of HIV infection (Figure 1.6b). When antiretroviral therapy is first offered to a community, not all HIV+ individuals will immediately begin drug therapy. If half of HIV + men start taking the drugs but if the amount of unprotected sex doubles $(i=1)$, then the number of new cases of HIV is predicted to double within the community following the beginning of antiretroviral therapy. This massive influx of new cases declines over time, however, as more HIV + men enter treatment. This decline results from the fact that ART lowers the viral load within 
(a)

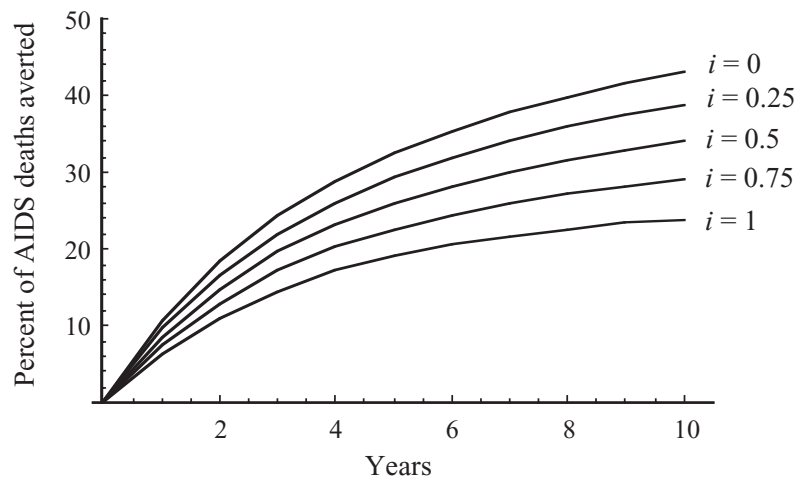

(b)

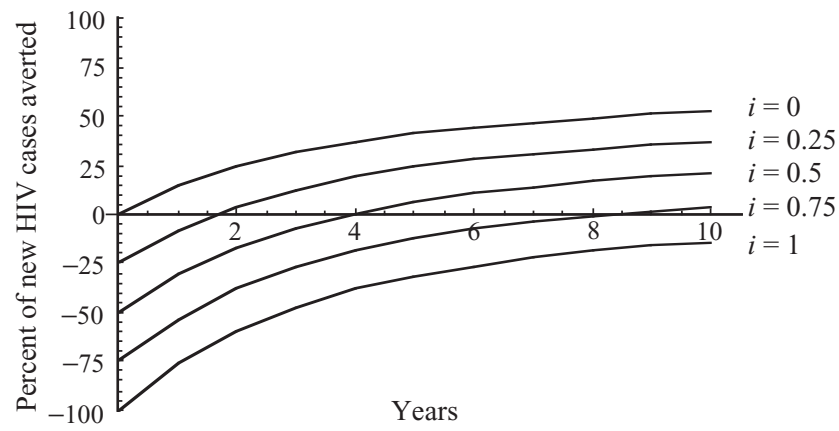

Figure 1.6: HIV, AIDS, and antiretroviral therapy. Percent reduction in the cumulative number of (a) AIDS deaths and (b) new HIV cases in the years following the onset of antiretroviral therapy (ART; modified from Blower et al. 2000). Each figure is drawn for various values of $i$, which measures the increase in risky behavior (unprotected sex) within the gay male community once ART becomes available, based on the equations developed in Box 2.5. A negative number on the $y$-axis implies that the cumulative number of cases increases not decreases.

the body, which decreases the probability that HIV will be transmitted through an exchange of bodily fluids. Over a ten-year period, the cumulative number of new cases of HIV is expected to be lower with ART than without as long as risky behavior increases by less than $80 \%$.

Blower et al. also modeled the evolution of drug resistance by including two more variables to describe individuals who are infected with resistant strains of HIV and are either untreated $\left(R_{U}\right)$ or treated $\left(R_{T}\right)$. They allowed drug resistance to evolve at a certain rate in HIV + individuals under ART and to affect most of the parameters in their model. The evolution of drug resistance led to a more pessimistic outlook, in terms of both the number of AIDS cases and the number of new infections, but the effect was relatively slight over the ten-year period that was considered. Over this time period, the rate at which HIV+ individuals enter into the treatment program and changes in risky behavior were much more important determinants of the forecasted number of HIV + cases and deaths due to AIDS.

By exploring the future of HIV and AIDS within the San Francisco gay community, the model of Blower et al. (2000) identified a major health care problem: if gay men are willing to take greater risks following the introduction of ART, many more new cases of HIV and many more deaths from AIDS will result than if safe sex practices remain in place. Unfortunately, recent trends in San Francisco have indicated that the level of risky behavior has increased dramatically in recent years (San Francisco Department of Public Health 2000). The percentage of gay men reporting sex with multiple partners and unprotected 
intercourse rose from $23 \%$ in 1994 to $47 \%$ in 2000 according to the STOP AIDS project. During the same period of time, the percentage of gay men reporting using condoms all of the time fell from $70 \%$ to $50 \%$. With such large increases in risky behavior, there has been an overall increase in HIV incidence, as predicted by the model of Blower et al. Among blood samples drawn at anonymous testing sites, the incidence of HIV increased from 1.3\% in 1997 to $4.2 \%$ in 1999 (San Francisco Department of Public Health 2000). Similar increases in incidence have been observed in Ontario, Canada (Calzavara et al. 2002).

\subsubsection{Estimating the Number of New Infections}

Preventing the spread of HIV requires the coordination of social, educational, and medical services. In many countries these services are in very limited supply and must be targeted to the populations most at risk of infection. The level of risk of an individual cannot, however, be directly measured and must be inferred. In contrast, it is relatively straightforward to determine who is already infected with HIV, as long as blood samples from representative individuals can be tested. But these infected individuals may have harbored HIV for many years and may not represent the demographic group most at risk of becoming newly infected. Long-term longitudinal studies, where thousands of uninfected individuals are regularly tested over many years, are the best way to determine risk factors. These studies are costly, however, and take many years before the data become available for use in prevention programs.

Alternatively, mathematical models can be used to estimate the number of new infections from data on the number of currently infected individuals. To determine the age group most at risk of infection, Williams et al. (2001) modeled changes in the HIV status of women aged 14 to 49 in the rural district of Hlabisa in South Africa. We analyze this model in the on-line supplementary material to Chapter 10 (see Sup. Mat. 10.2). HIV has been spreading in South Africa at an exponential rate since the early 1980s, with the number of HIVinfected people doubling approximately every two years. In Hlabisa, a broad survey of HIV status was possible because almost all pregnant women (95\%) in this region attend hospitals or clinics for prenatal care. During 1998, 3163 of these women gave blood samples that were tested for HIV. The prevalence of HIV among these women (i.e., the fraction of women that were HIV+) is shown in Figure 1.7a.

Williams et al. (2001) developed a model to estimate the risk of contracting HIV (see the on-line Supplementary Material to Chapter 10, Sup. Mat. 10.2). The more specific aim of the study was to estimate the probability per year that an uninfected woman in Hlabisa becomes newly infected with HIV, for women of various ages (this probability is known as the incidence of HIV). To estimate this unknown risk, their model related the probability of contracting HIV and the death rate from AIDS to the changes that should occur from year to year in the fraction of infected women. This study uses a model in a fundamentally different way from the previous examples. In all three previous examples, models were used to project into the future, describing how a population (of cells or of individuals) was expected to change over time given various parameters. In this 
(c) Copyright, Princeton University Press. No part of this book may be distributed, posted, or reproduced in any form by digital or mechanical means without prior written permission of the publisher.

(a)

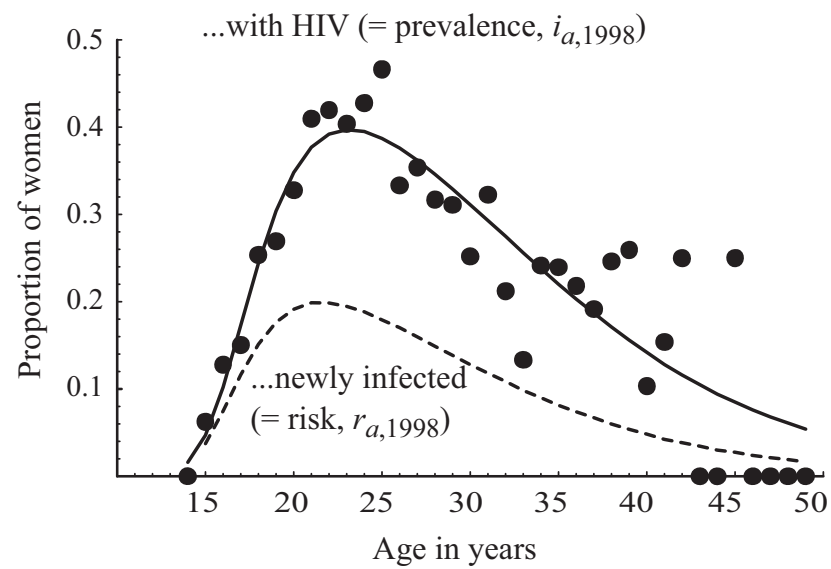

(b)

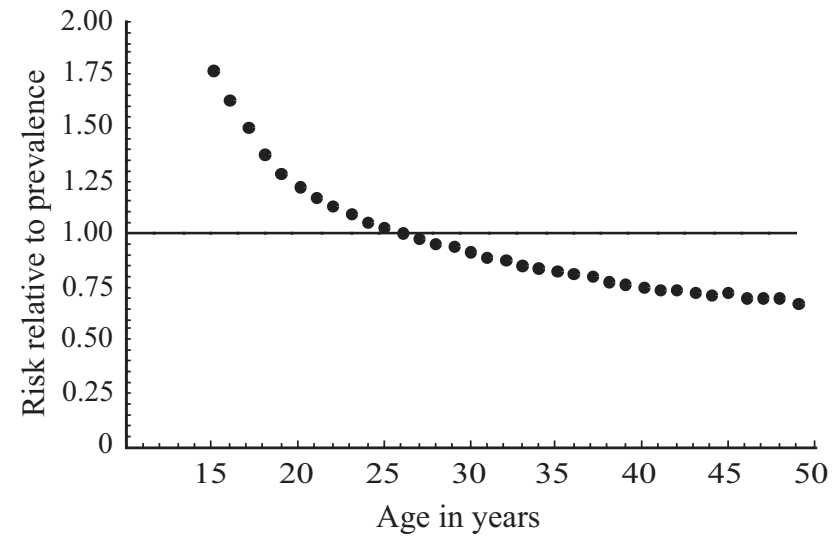

Figure 1.7: The impact of HIV on the population of pregnant women from Hlabisa, South Africa.

(a) Shown are the proportion of women who were $\mathrm{HIV}+$ in 1998 (prevalence, given by dots) and the fraction of HIV- women who contract HIV annually (risk, given by the dashed curve, from equation S10.2.8 in Sup. Mat. 10.2). The solid curve is fit to the prevalence data using polynomial smoothing (see Williams et al., 2001). (b) The age distribution of risk is compared to the age distribution of HIV+ prevalence. Each dot represents the probability that a woman who newly acquires HIV is of age $a$

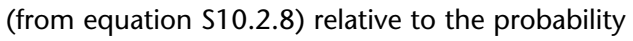
that a woman who has HIV is of age $a$. This figure tells us which age classes are more at risk of acquiring HIV than expected based on who has HIV (points above one; solid line).

case, however, Williams et al. knew the state of the population in 1998, but they wanted to infer something about the parameters that might have led to that pattern. Thus, models can be used both to generate predictions about the future and to make inferences about the past.

Figure 1.7a shows data from 1998 on the number of women of age $a$ infected with HIV (prevalence $P_{a, 1998}$ ) and compares this to the inferred risk of infection from HIV as a function of age (risk $r_{a, 1998}$ ) based on the model. The peak age of risk (21-22 years) is very nearly equal to the peak prevalence (23 years). This indicates that most HIV + women attending hospitals and clinics in Hlibasi became infected within the last couple of years. Williams et al. (2001) noted that the shortness of the period since infection most likely reflects the fact that HIV is spreading rapidly through the region, so that many infections are newer than would be expected if HIV were well established within the population (endemic). Another important result is that the current prevalence data do not give a very good picture of who is most at risk. This is illustrated in Figure 1.7b, which gives the risk of contracting HIV for women at each age relative to that expected from the prevalence data. This figure illustrates that teenagers are up to 75\% more likely to contract HIV than would be expected from the low prevalence of HIV among younger women. In the absence of a long-term longitudinal 
study, the estimates provided by Williams et al. (2001) help to assess the risk of HIV infection as a function of age and can be used to better target programs designed to halt the spread of HIV in South Africa.

\subsection{Concluding Message}

Through reading this book, we hope that you will come to appreciate just how useful mathematical modeling can be in biology. Models can help to guide a scientist's intuition about how various processes interact; they can point out logical flaws in an argument; they can identify testable hypotheses, generate key predictions, and suggest appropriate experiments; and they can reshape fields by providing new ways of thinking about a problem.

But mathematical models also have their limitations. The results of an analysis are only as interesting as the biological questions motivating a model. And even if a scientist has identified an interesting question, it may turn out that a model addressing the question is hopelessly complicated and can be solved only by making a series of assumptions, some of which are dubious. Finally, models, by themselves, can only tell us what is possible. Models can tell us, for example, how HIV levels within the body or HIV incidence within a population might change over time. But without data, collected in the field or the lab, mathematical models can never tell us what has happened or what is happening. Thus, it would be foolish to promote mathematical biology above other areas in biology. Equally, it would be foolish to avoid mathematics altogether. Science will progress faster and further by a marriage of mathematical and empirical biology. This marriage will be even more successful if more biologists can use math, when needed, to advance their own research goals. It is toward this end that we devote this book.

\section{Further Reading}

For general information about the immune system and the evolution and ecology of infectious diseases, see

- Frank, S. A. 2002. Immunology and Evolution of Infectious Diseases. Princeton University Press, Princeton, N.J.

Further information concerning the life cycle, health impact, and societal implications of HIV is available through the links on the book website (http://press.princeton.edu/ titles/8458.html) and at www.zoology.ubc.ca/biomath. Also, see

- Moore, R. D. and Bartlett, J. G. (1998) Improving HIV therapy. Scientific American 279: 84-93.

\section{References}

Bacchetti, P., and A. R. Moss. 1989. Incubation period of AIDS in San Francisco. Nature 338:251-253 
Bacchetti, P., D. Osmond, R. E. Chaisson, S. Dritz, G. W. Rutherford, L. Swig, and A. R. Moss. 1988. Survival patterns of the first 500 patients with AIDS in San Francisco. J. Infect. Dis 157:1044-1047.

Blower, S. M., H. B. Gershengorn, and R. M. Grant. 2000. A tale of two futures: HIV and antiretroviral therapy in San Francisco. Science 287:650-654.

Calzavera, L., et al. 2002. Increases in HIV incidence among men who have sex with men undergoing repeat diagnostic HIV testing in Ontario, Canada. AIDS 16:1655-1661.

Dimitrov, D. S., R. L. Willey, H. Sato, L. J. Chang, R. Blumenthal, and M. A. Martin. 1993. Quantitation of human immunodeficiency virus type 1 infection kinetics. J. Virol. 67:2182-2190.

Fauci, A. S., G. Pantaleo, S. Stanley, and D. Weissman. 1996. Immunopathogenic mechanisms of HIV infection. Ann. Intern. Med. 124:654-663.

Funk, G. A., M. Fischer, B. Joos, M. Opravil, H. F. Gunthard, B. Ledergerber, and S. Bonhoeffer. 2001. Quantification of in vivo replicative capacity of HIV-1 in different compartments of infected cells. J. Acquired Immune Defic. Syndr. 26:397-404.

Hansson, T., and J. Aqvist. 1995. Estimation of binding free energies for HIV proteinase inhibitors by molecular dynamics simulations. Protein Eng. 8:1137-1144.

Ho, D. D., A. U. Neumann, A. S. Perelson, W. Chen, J. M. Leonard, and M. Markowitz. 1995. Rapid turnover of plasma virions and CD4 lymphocytes in HIV-1 infection. Nature 373:123-126.

Jacquez, J. A., J. S. Koopman, C. P. Simon, and I. M. Longini, Jr. 1994. Role of the primary infection in epidemics of HIV infection in gay cohorts. J. Acquired Immune Defic. Syndr. 7:1169-1184.

Joint United Nations Programme on HIV/AIDS. 2004a. AIDS epidemic update, December 2004. UNAIDS, Geneva, Switzerland.

Joint United Nations Programme on HIV/AIDS. 2004b. UNAIDS 2004 Report on the global AIDS epidemic. UNAIDS, Geneva, Switzerland.

Koopman, J. S., J. A. Jacquez, G. W. Welch, C. P. Simon, B. Foxman, S. M. Pollock, D. Barth-Jones, A. L. Adams, and K. Lange. 1997. The role of early HIV infection in the spread of HIV through populations. J. Acquired Immune Defic. Syndr. Hum. Retrovirol. 14:249-258.

Koup, R. A., J. T. Safrit, Y. Cao, C. A. Andrews, G. McLeod, W. Borkowsky, C. Farthing, and D. D. Ho. 1994. Temporal association of cellular immune responses with the initial control of viremia in primary human immunodeficiency virus type 1 syndrome. J. Virol. 68:4650-4655.

Kroeger Smith, M. B. et al. 1995. Molecular modeling studies of HIV-1 reverse transcriptase nonnucleoside inhibitors: Total energy of complexation as a predictor of drug placement and activity. Protein Sci. 4:2203-2222.

Levin, B. R., J. J. Bull, and F. M. Stewart. 1996. The intrinsic rate of increase of HIV/AIDS: epidemiological and evolutionary implications. Math. Biosci. 132:69-96.

Lloyd, A. L. 2001. The dependence of viral parameter estimates on the assumed viral life cycle: Limitations of studies of viral load data. Proc. R. Soc., Seri. B 268: 847-854.

Markgren, P. O., M. T. Lindgren, K. Gertow, R. Karlsson, M. Hamalainen, and U. H. Danielson. 2001. Determination of interaction kinetic constants for HIV-1 protease inhibitors using optical biosensor technology. Anal. Biochem. 291:207-218. 
Nowak, M. A., S. Bonhoeffer, C. Loveday, P. Balfe, M. Semple, S. Kaye, M. Tenant-Flowers, and R. Tedder. 1995. HIV results in the frame. Results confirmed. Nature 375:193.

Perelson, A. S., A. U. Neumann, M. Markowitz, J. M. Leonard, and D. D. Ho. 1996. HIV-1 dynamics in vivo: virion clearance rate, infected cell life-span, and viral generation time. Science 271:1582-1586.

Perrin, L., and S. Yerly. 1997. Clinical presentation, virological features, and treatment perspectives in primary HIV-1 infection. AIDS Clin. Rev.: 67-92.

Phillips, A. N. 1996. Reduction of HIV concentration during acute infection: Independence from a specific immune response. Science 271:497-499.

Revelle, M. 1995. Progress in blood supply safety. FDA Consum. 29:21-124.

San Francisco Department of Public Health. 2000. HIV/AIDS Epidemiology Annual Report 2000 (http://www.dph.sf.ca.us/Reports/HlthAssess.htm). San Francisco, CA.

Schacker, T., A. C. Collier, J. Hughes, T. Shea, and L. Corey. 1996. Clinical and epidemiologic features of primary HIV infection. Ann. Intern Med. 125:257-264.

Schmitz, J. E., M. J. Kuroda, S. Santra, V. G. Sasseville, M. A. Simon, M. A. Lifton, P. Racz, K. Tenner-Racz, M. Dalesandro, B. J. Scallon, J. Ghrayeb, M. A. Forman, D. C. Montefiori, E. P. Rieber, N. L. Letvin, and K. A. Reimann. 1999. Control of viremia in simian immunodeficiency virus infection by CD8+ lymphocytes. Science 283:857-860.

Stafford, M. A., L. Corey, Y. Cao, E. S. Daar, D. D. Ho, and A. S. Perelson. 2000. Modeling plasma virus concentration during primary HIV infection. J. Theor. Biol. 203: $285-301$.

Wei, X. et al. 1995. Viral dynamics in human immunodeficiency virus type 1 infection. Nature 373:117-122.

Williams, B., E. Gouws, D. Wilkinson, and S. A. Karim. 2001. Estimating HIV incidence rates from age prevalence data in epidemic situations. Stat. Med. 20:2003-2016. 